\title{
Probing Non-Uniform Adsorption in Multicomponent Metal-Organic Frameworks via Segmental Dynamics by Solid-State Nuclear Magnetic Resonance
}

Hanxi Guan ${ }^{1}$, Jiachen $\mathrm{Li}^{2}$, Tianyou Zhou ${ }^{3}$, Zhenfeng Pang ${ }^{1}, \mathrm{Yao} \mathrm{Fu}^{1}$, Joel Cornelio ${ }^{3}$, Qi Wang ${ }^{2 *}$, Shane G. Telfer ${ }^{3 *}$, Xueqian Kong ${ }^{1 *}$.

${ }^{1}$ Center for Chemistry of High-Performance \& Novel Materials, Department of Chemistry, Zhejiang University, Hangzhou 310027, People's Republic of China.

${ }^{2}$ Department of Chemistry, Zhejiang University, Hangzhou 310027, People’s Republic of China.

${ }^{3}$ MacDiarmid Institute for Advanced Materials and Nanotechnology, School of Fundamental Sciences, Massey University, Palmerston North 4442, New Zealand.

\section{AUTHOR INFORMATION}

\section{Corresponding Author}

Qi Wang: $\quad$ qiwang@zju.edu.cn

Shane G. Telfer: $\quad$ s.telfer@massey.ac.nz

Xueqian Kong: $\quad$ kxq@zju.edu.cn. 


\section{CONTENTS}

1. Experimental Section

1.1 Synthesis of Deuterated MUF-77

1.2 Adsorption Methods

1.3 Powder X-Ray Diffraction

$1.4{ }^{1} \mathrm{H}$ solution-state NMR

$1.5^{2} \mathrm{H}$ Lineshape Simulation

1.6 Spin-lattice Relaxation Measurement

1.7 Force Field and Simulation Details of Molecular Dynamics Simulation

2. Supplementary Figures and Tables

2.1 Basic Characterizations of MUF-77

2.2 Dynamics and Host-Guest Interaction Study 


\section{Experimental Section}

\subsection{Synthesis of Deuterated MUF-77}

The synthesis and activation of deuterated MUF-77-M and MUF-77-D was performed as earlier reported, ${ }^{1}$ with deuterated $\mathrm{H}_{2}$ bdc and $\mathrm{H}_{2}$ bpdc as reactants, respectively.

MUF-77-M with d4-bdc: A suspension of $\mathrm{H}_{3} \mathrm{hmtt}(18.0 \mathrm{mg}, 32.2 \mu \mathrm{mol}), \mathrm{d} 4-\mathrm{H}_{2} \mathrm{bdc}(6.2 \mathrm{mg}$, $37.06 \mu \mathrm{mol}), \mathrm{H}_{2} \mathrm{bpdc}(12.1 \mathrm{mg}, 48.3 \mu \mathrm{mol}), \mathrm{Zn}\left(\mathrm{NO}_{3}\right)_{2}\left(\mathrm{H}_{2} \mathrm{O}\right)_{4}(58.9 \mathrm{mg}, 225.5 \mu \mathrm{mol}), \mathrm{DEF}$ (diethyl formamide, $4 \mathrm{~mL})$ and water $(140 \mu \mathrm{L})$ was sonicated for 30 min before being heated in an $85^{\circ} \mathrm{C}$ isothermal oven overnight to obtain pale yellow crystals of d4-MUF-77-M. The mother liquor was decanted when it was still warm and replaced with anhydrous DMF (dimethylformamide). After washing with dried DCM (dichloromethane), the sample was activated under vacuum at $80{ }^{\circ} \mathrm{C}$ for 12 hours.

MUF-77-M with d8-bpdc: A suspension of $\mathrm{H}_{3} \mathrm{hmtt}(18.0 \mathrm{mg}, 32.2 \mu \mathrm{mol}), \mathrm{H}_{2}$ bdc (6.2 mg, 37.06 $\mu \mathrm{mol})$, d8- $\mathrm{H}_{2}$ bpdc (12.1 mg, $\left.48.3 \mu \mathrm{mol}\right), \mathrm{Zn}\left(\mathrm{NO}_{3}\right)_{2}\left(\mathrm{H}_{2} \mathrm{O}\right)_{4}(58.9 \mathrm{mg}, 225.5 \mu \mathrm{mol}), \mathrm{DEF}(4 \mathrm{~mL})$ and water $(140 \mu \mathrm{L})$ was sonicated for 30 min before being heated in an $85{ }^{\circ} \mathrm{C}$ isothermal oven overnight to obtain pale yellow crystals of d8-MUF-77-M. The mother liquor was decanted when it was still warm and replaced with anhydrous DMF. After washing with dried DCM, the sample was activated under vacuum at $80^{\circ} \mathrm{C}$ for 12 hours.

MUF-77-D with d8-bpdc: A suspension of $\mathrm{H}_{3}$ hdtt (42.5 mg, $\left.32.2 \mu \mathrm{mol}\right), \mathrm{H}_{2}$ bdc (5.30 mg, 31.7 $\mu \mathrm{mol}), \mathrm{d}_{8}-\mathrm{H}_{2} \operatorname{bpdc}(10.4 \mathrm{mg}, 41.6 \mu \mathrm{mol}), \mathrm{Zn}\left(\mathrm{NO}_{3}\right)_{2}\left(\mathrm{H}_{2} \mathrm{O}\right)_{4}(58.9 \mathrm{mg}, 225.5 \mu \mathrm{mol}), \mathrm{DEF}(4 \mathrm{~mL})$ and 
water $(140 \mu \mathrm{L})$ was sonicated for $30 \mathrm{~min}$ before being heated in an $85^{\circ} \mathrm{C}$ isothermal oven overnight to obtain pale yellow crystals of d8-MUF-77-D. The aftertreatment was the same as d8-MUF-77M.

\subsection{Adsorption Methods}

Methanol adsorption: $40 \mathrm{mg}$ d8-MUF-77-M and d8-MUF-77-D powders were exposed to methanol vapor under room temperature at $1 \mathrm{~atm}$ for 36 hours to reach equilibrium, respectively. Then the sample was transferred into a $3.2 \mathrm{~mm}$ Bruker $\mathrm{ZrO}_{2}$ rotor and sealed for SSNMR measurement.

DMA adsorption: $40 \mathrm{mg}$ d8-MUF-77-M and 40mg d8-MUF-77-D were soaked for 24 hours in $15 \mathrm{ml}$ solution with the volume ratio of $\mathrm{N}, \mathrm{N}$-dimethylaniline: methanol $=1: 2$, Then the crystals were washed twice with methanol and dried under vacuum at $70{ }^{\circ} \mathrm{C}$ to remove methanol.

AO adsorption: $115 \mathrm{mg}$ d8-MUF-77-M were soaked for 24 hours in a DMF solution (250 mM, $20 \mathrm{ml}$ ) of acridine orange and then washed twice with dichloromethane and dried under vacuum to produce the acridine orange loaded d8-MUF-77-M.

\subsection{Powder X-ray Diffraction}

Powder X-ray diffraction experiments were carried out on a Rigaku Spider X-ray diffractometer with $\mathrm{Cu} \mathrm{K} \alpha$ radiation (Rigaku MM007 microfocus rotating-anode generator), monochromated and focused with high-flux Osmic multilayer mirror optics, and a curved image plate detector. 


\subsection{Solution-state ${ }^{1} \mathrm{H}$ NMR}

The sample was washed with dry $\mathrm{CH}_{2} \mathrm{Cl}_{2}$ or acetone and desolvated in vacuo. $0.60 \mathrm{~mL}$ of $\mathrm{DCl} / \mathrm{DMSO}-\mathrm{d}_{6}$ (dimethylsulfoxide) $(10 \mu \mathrm{L} / 600 \mu \mathrm{L})$ solution was used to digest around $3 \mathrm{mg}$ of MOF. Then ${ }^{1} \mathrm{H}$ NMR spectrum was acquired of the resulting clear solution of the dissolved framework.

\section{$1.5{ }^{2} \mathrm{H}$ Lineshape Simulation}

${ }^{2} \mathrm{H}$ lineshape simulation were carried out as a multi-site-jump model using EXPRESS ${ }^{2}$ package running in MATLAB software. ${ }^{3}$ The ${ }^{2} \mathrm{H}$ powder pattern lineshapes are sensitive to molecular motions with correlation times of the order $10^{-4}$ to $10^{-8} \mathrm{~s}$, which corresponds to motional correlation times often found in solids. ${ }^{2} \mathrm{H}$ SSNMR has been extensively used in motional studies. ${ }^{4-6}$ In this work, the quadrupolar coupling constant was set to $180 \mathrm{kHz}$ and the asymmetry parameter was set to 0.04 according to the experimental lineshape under $245 \mathrm{~K}$ temperature and the value of $s p^{2} \mathrm{C}$ D bond described in literature. ${ }^{7-10}$

\subsection{Spin-lattice Relaxation Time Measurement}

${ }^{2} \mathrm{H}$ spin-lattice relaxation time $\left(\mathrm{T}_{1}\right)$ measurements were obtained by inversion recovery quadrupolar echo sequence under $5 \mathrm{k}$ to $10 \mathrm{kHz}$ MAS condition. The ${ }^{2} \mathrm{H} \mathrm{T}_{1}$ relaxation times for the d8-MUF-77-M range from 15 and $90 \mathrm{~ms}$ between $323 \mathrm{~K}$ and $275 \mathrm{~K}$, while for d8-MUF-77-D range from 15 to $115 \mathrm{~ms}$ in the same temperature range. 
The relationship between $T_{1}$ and temperature is described by a general formalism: ${ }^{11}$

$$
T_{1}=\frac{3 \pi^{2} C_{Q}{ }^{2}}{2}\left[J_{1}(\omega)+4 J_{2}(2 \omega)\right]=\frac{3 \pi^{2} C_{Q}{ }^{2}}{2}\left[\frac{\tau_{c}}{1+\omega^{2} \tau_{c}^{2}}+4 \frac{\tau_{c}}{1+4 \omega^{2} \tau_{c}^{2}}\right]
$$

$\tau_{c}$ is the correlation time of a particular type of dynamic motion. The rate of motion obeys the Arrhenius's Law:

$$
\frac{1}{\tau_{c}}=\frac{1}{\tau_{0}} e^{-\frac{E_{a}}{R T}}
$$

In the fast limit, $\omega^{2} \tau_{c}{ }^{2} \ll 1$

The formalism can be simplified as $T_{1}=\frac{3 \pi^{2} C_{Q}{ }^{2}}{2} \times 5 \tau_{c}$.

In such condition, $\frac{1}{T_{1}} \propto \frac{1}{\tau_{c}}=\frac{1}{\tau_{0}} e^{-\frac{E_{a}}{R T}}$.

We can calculate the rotational barrier of dynamics from the slope of the $\ln \left(1 / T_{1}\right)$ versus $1 / T$.

\subsection{Force Field and Simulation Details of Molecular Dynamics Simulation}

The MUF-77-M and MUF-77-D were mixed with methanol $\left(\mathrm{CH}_{3} \mathrm{OH}\right)$, N,N-dimethylaniline (DMA, $\left.\mathrm{C}_{8} \mathrm{H}_{11} \mathrm{~N}\right)$ and Acridine Orange $\left(\mathrm{AO}, \mathrm{C}_{17} \mathrm{H}_{19} \mathrm{~N}_{3}\right)$ shown in Table S1, respectively. Different number of solvent molecules were added depending on their sizes and the experimental order. All MD simulations were performed using the GROMACS-5.0.7 package with a time step of $2 \mathrm{fs} .{ }^{12}$ The forcefield parameters of one part of MUF-77-M, MUF-77-D and guest molecules, including bpdc, bdc, ME, DMA and AO were obtained from the Automated Topology Builder (ATB) server. ${ }^{13}$ The topologies of hmtt of MUF-77-M were also generated using ATB server, and the 
charge of hmtt were calculated using restrained electrostatic potential (RESP) method at the HF/6$31 \mathrm{G}+$ level in Gaussian 03. ${ }^{14}$ The forcefield parameters of $\mathrm{Zn}_{4} \mathrm{O}$ clusters of MUF-77-M and MUF77-D were taken from Tafipolsky's paper. ${ }^{15}$ In all simulations, the Lennard-Jones (LJ) parameters of atoms were derived from the GROMOS96 force field. ${ }^{16}$ All atoms of MUF-77 were frozen during simulation, except for the benzene ring atom of bpdc, as the experimental results shows they jump quickly. The long-range electrostatic interaction were calculated based on the Particle mesh Ewald algorithm ${ }^{17}$, and the cutoff was set to be $1.3 \mathrm{~nm}$. The $\mathrm{LJ}$ potentials of cross interaction between MUF-77-M and different adsorbate molecules were obtained by Lorentz-Berthelot rule. The periodic boundary conditions $(\mathrm{PBC})$ was used in all three directions. After energy minimization and pre-equilibration of the system, the production runs at the constant temperature $(300 \mathrm{~K})$ controlled by Berendsen algorithm ${ }^{18}$. Each simulation was conducted in 20 ns $N V T$ ensemble, where the last 5 ns were used for analysis. 
Table S1. The parameters used for MD simulations.

\begin{tabular}{|c|c|c|c|c|c|}
\hline \multicolumn{6}{|c|}{ Number of compartments in simulated MUF-77-M/D } \\
\hline $\mathrm{hmtt} / \mathrm{hdtt}$ & bpdc & bdc & $\mathrm{Zn}_{4} \mathrm{O}$ cluster & $\mathrm{Zn}_{4} \mathrm{O}(\mathrm{h}$ & pdc) $)_{1 / 2}(\mathrm{bdc})_{1 / 2}$ \\
\hline 216 & 81 & 81 & 162 & & \\
\hline \multicolumn{6}{|c|}{ The loading capacity of adsorbates } \\
\hline methanol $^{+}$ & Conc. ${ }^{*}$ & $\mathrm{DMA}^{+}$ & Conc. ${ }^{*}$ & $\mathrm{AO}^{+}$ & Conc. ${ }^{*}$ \\
\hline 1000 & 6.2 & 500 & 3.1 & 100 & 0.6 \\
\hline 1500 & 9.3 & 750 & 4.6 & 200 & 1.2 \\
\hline 2000 & 12.3 & 1000 & 6.2 & 400 & 2.5 \\
\hline
\end{tabular}

${ }^{+}$. The number of adsorbates used in the simulation system.

* Conc. $=\frac{N u m_{\text {adsorbates }}}{\text { Num }_{Z n 4 O(h m t t) 4 / 3(b p d c) 1 / 2(b d c) 1 / 2}}$ or $\frac{N u m_{\text {adsorbates }}}{N u m_{Z n 4 O(h d t t) 4 / 3(b p d c) 1 / 2(b d c) 1 / 2}}$ 


\section{Supplementary Figures and Tables}

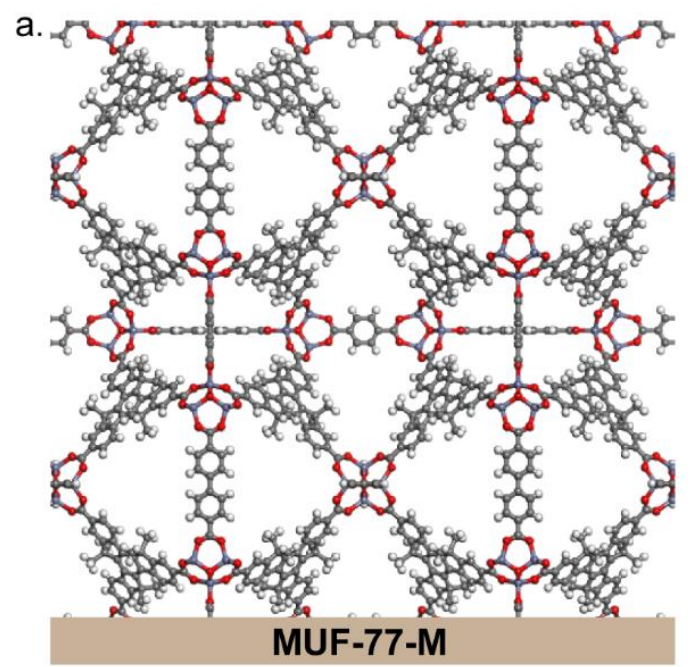

c.

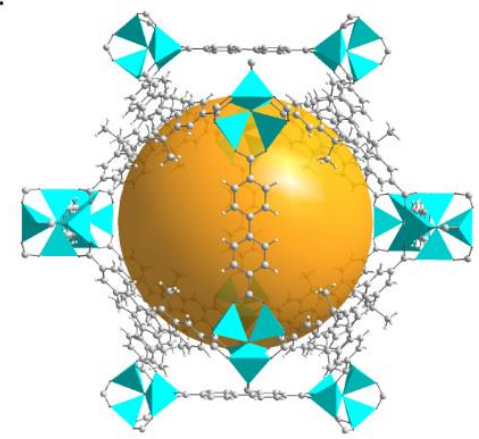

d.

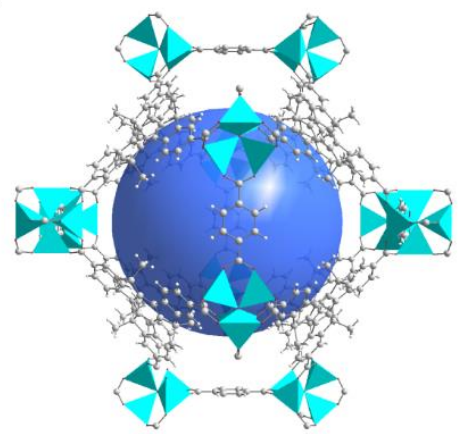

b.

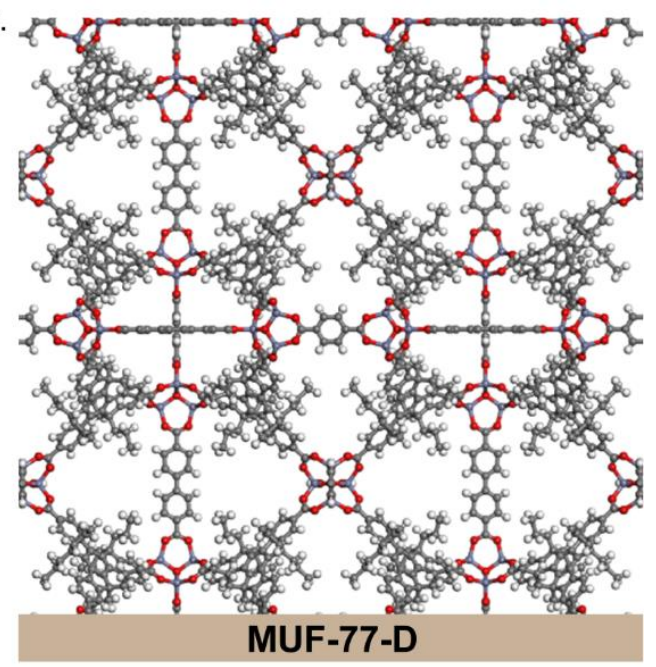

e.

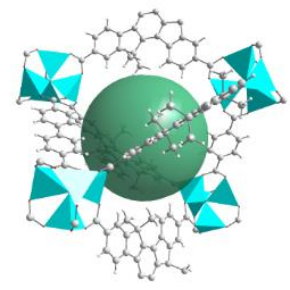

Figure S1. The crystal structures of (a) MUF-77-M and (b) MUF-77-D. The decyl chain in MUF77-D is highly mobile that only three carbons can be defined in the crystal file. Views of (c) large, (d) medium, (e) small pores in MUF-77-M. The spheres colored in orange, blue and green represent the void space of pores. The radius of the spheres is $1.1,1.0$ and $0.55 \mathrm{~nm}$, respectively., corresponding to volumes of 5.57, 4.18 and $0.7 \mathrm{~nm}^{3}$. 


\subsection{Basic Characterizations of MUF-77}
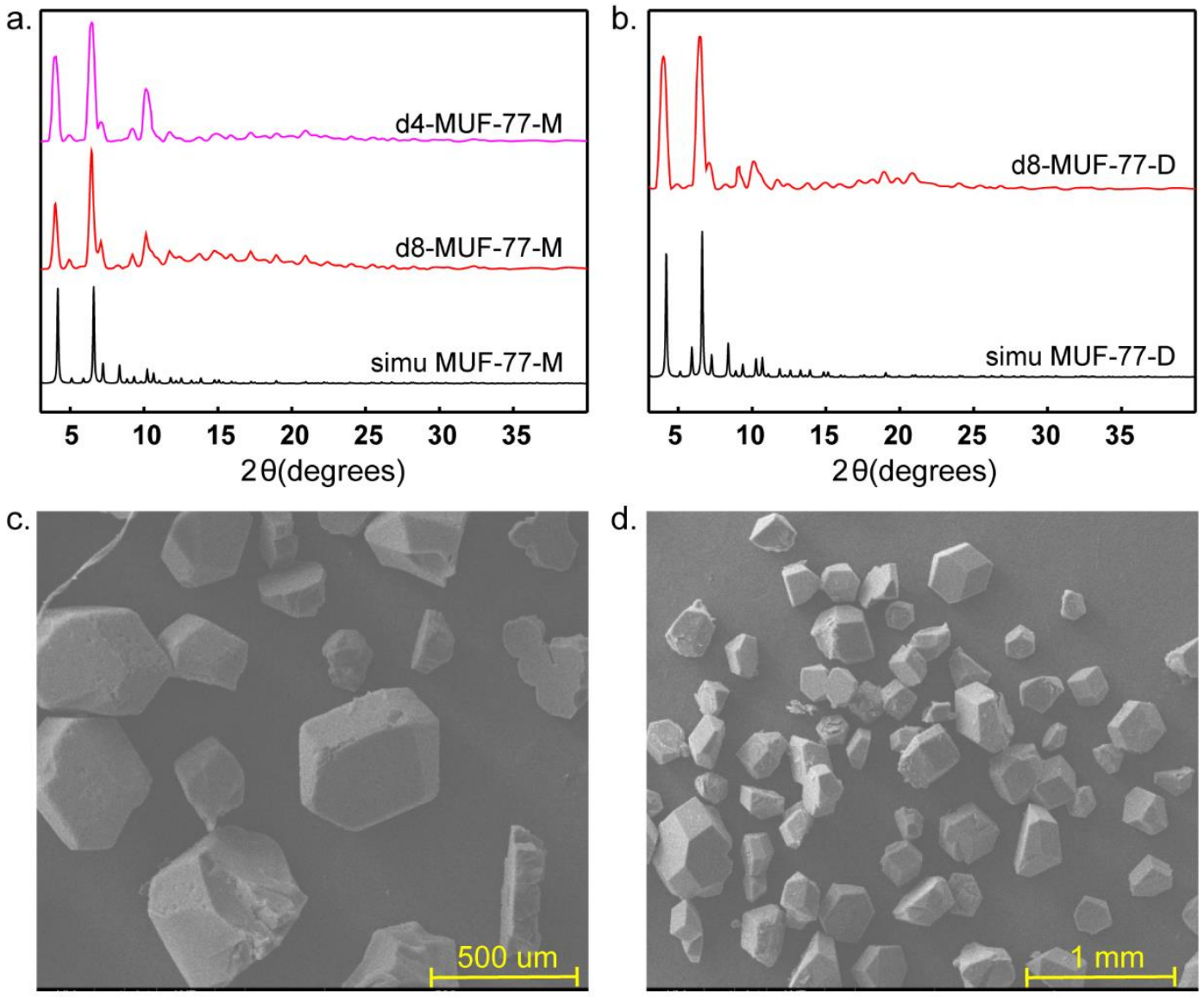

Figure S2. Powder XRD patterns of (a) deuterated MUF-77-M and (b) d8-MUF-77-D. SEM pictures of (c) d8-MUF-77-M and (d) d4-MUF-77-M show that the sizes of d8-MUF-77-M and d4-MUF-77-M vary from $100 \mu \mathrm{m}$ to over $500 \mu \mathrm{m}$. 


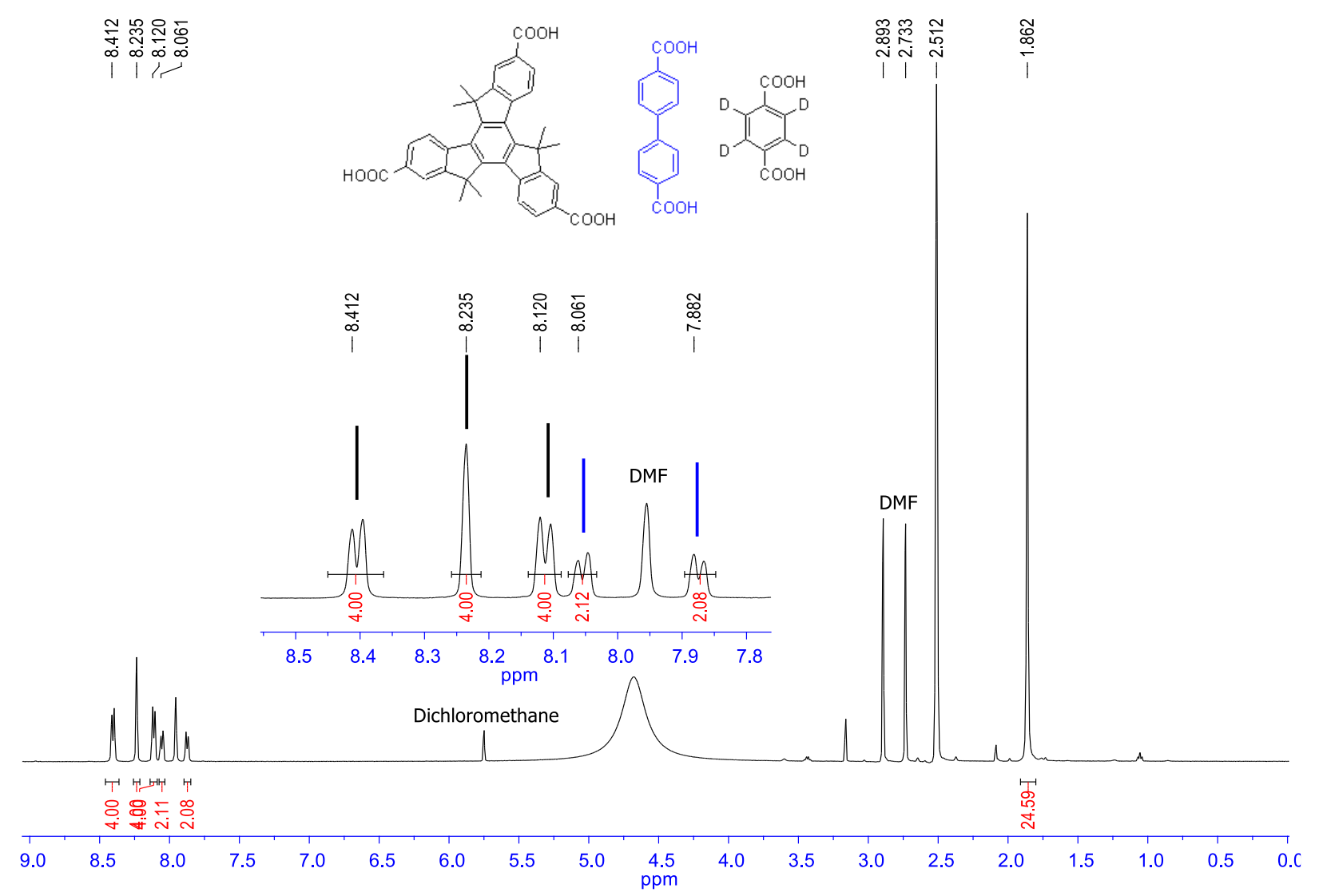

Figure S3. ${ }^{1} \mathrm{H}$ NMR of digested d4-MUF-77-M. The range from 7.8 to $8.5 \mathrm{ppm}$ is magnified in the figure. The peaks labeled by blue lines $(8.061 \mathrm{ppm}, 7.882 \mathrm{ppm})$ correspond to the protons of bpdc, while black ones correspond to the protons of hmtt. The chemical shift and the peak integral of each peak are shown in black and red numbers, respectively. 


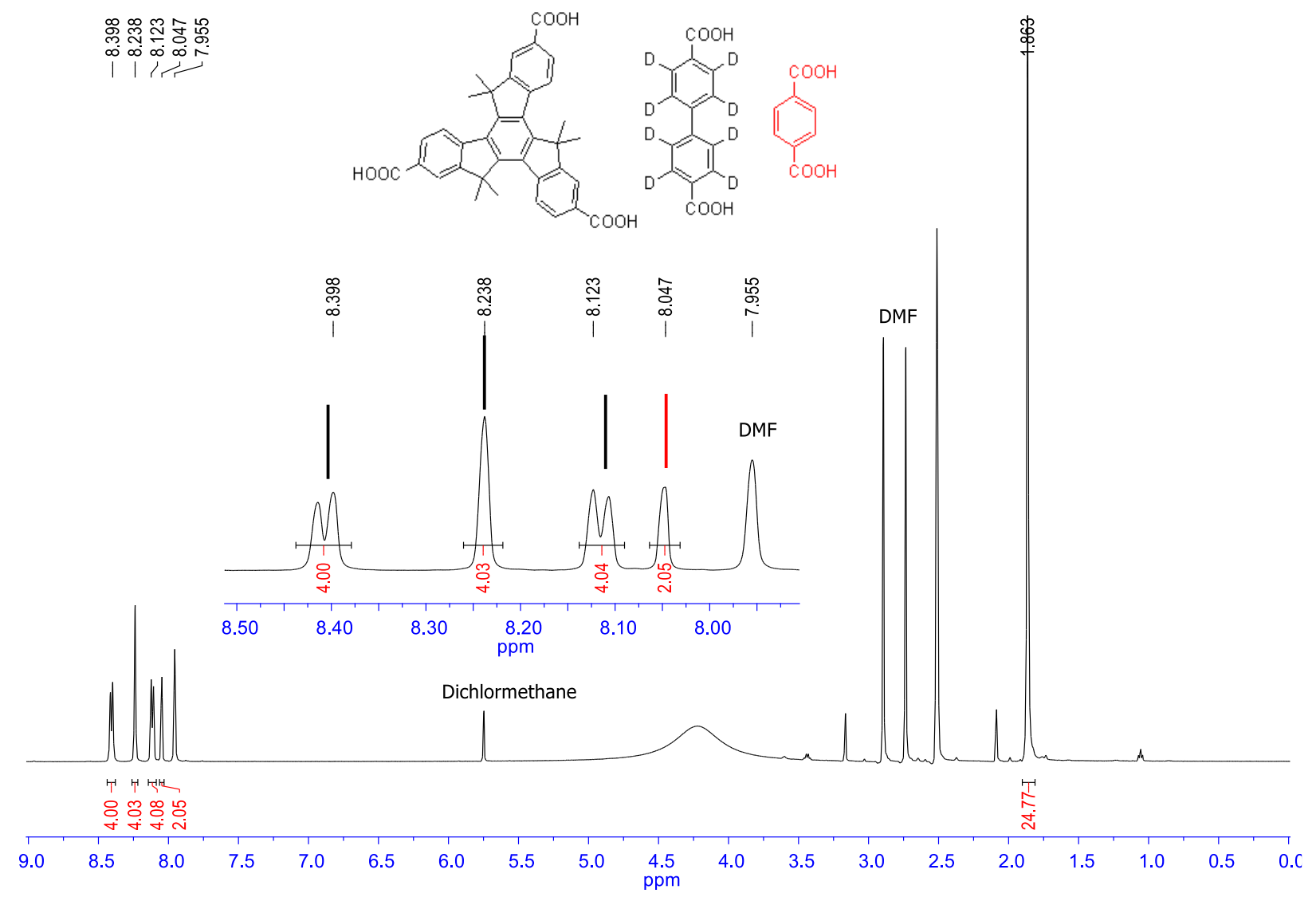

Figure S4. ${ }^{1} \mathrm{H}$ NMR of digested d8-MUF-77-M. The range from 7.90 to $8.50 \mathrm{ppm}$ is magnified in the figure. The peak labeled by red line $(8.047 \mathrm{ppm})$ corresponds to the protons of bdc, while black ones correspond to the protons of hmtt. The chemical shift and the peak integral of each peak are shown in the figure in black and red numbers, respectively. 


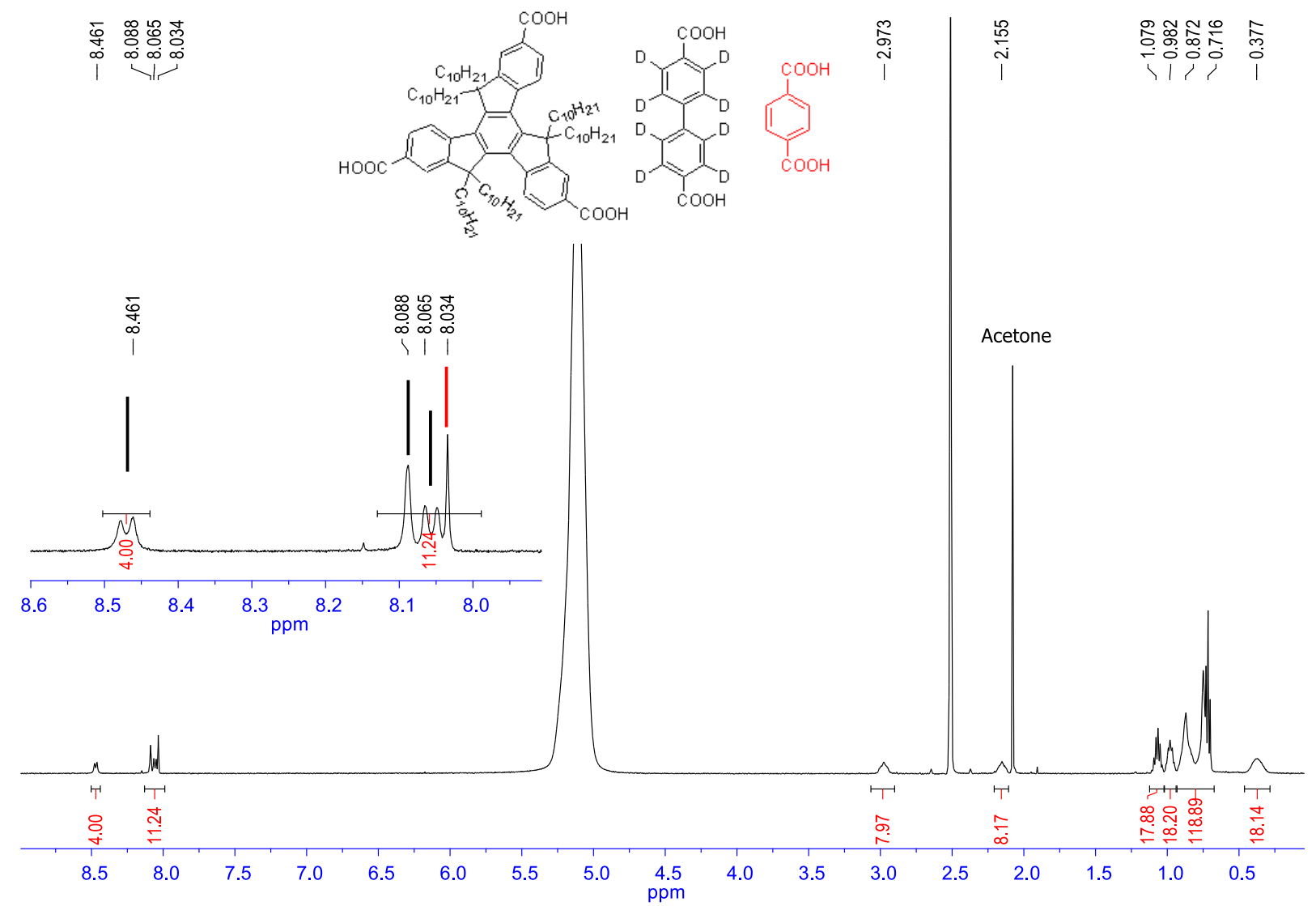

Figure S5. ${ }^{1} \mathrm{H}$ NMR of digested d8-MUF-77-D. The range from 7.90 to $8.60 \mathrm{ppm}$ is magnified in the figure. The peak labeled by red line $(8.034 \mathrm{ppm})$ corresponds to the protons of bdc, while black ones correspond to the protons of hmtt. The chemical shift and the peak integral of each peak are shown in black and red numbers, respectively. 


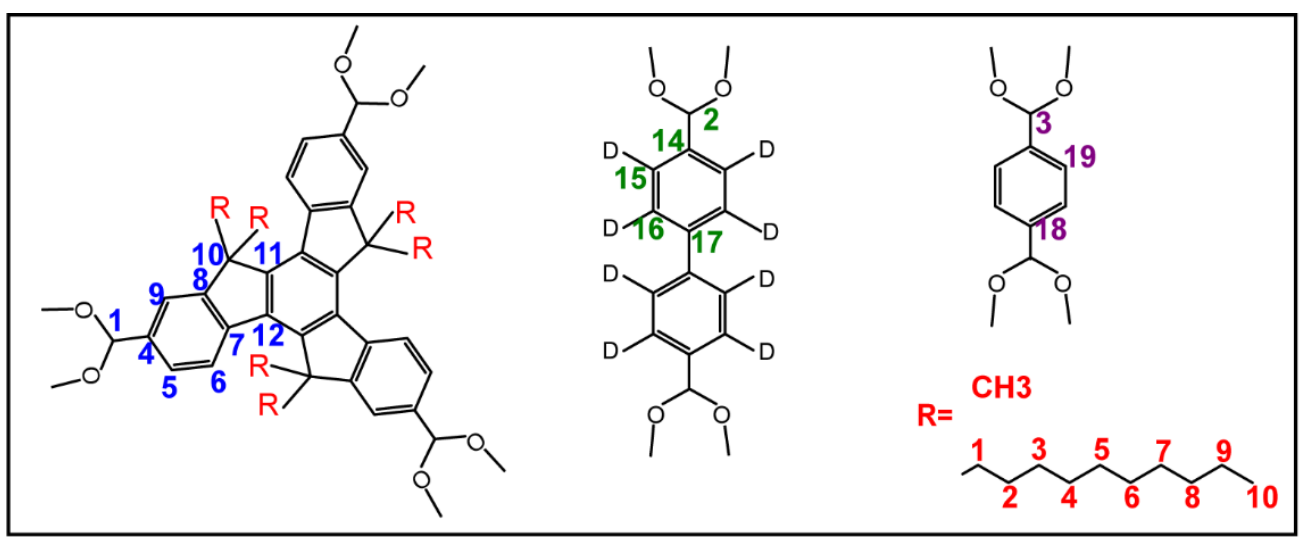

d8-MUF-77-M HPDEC

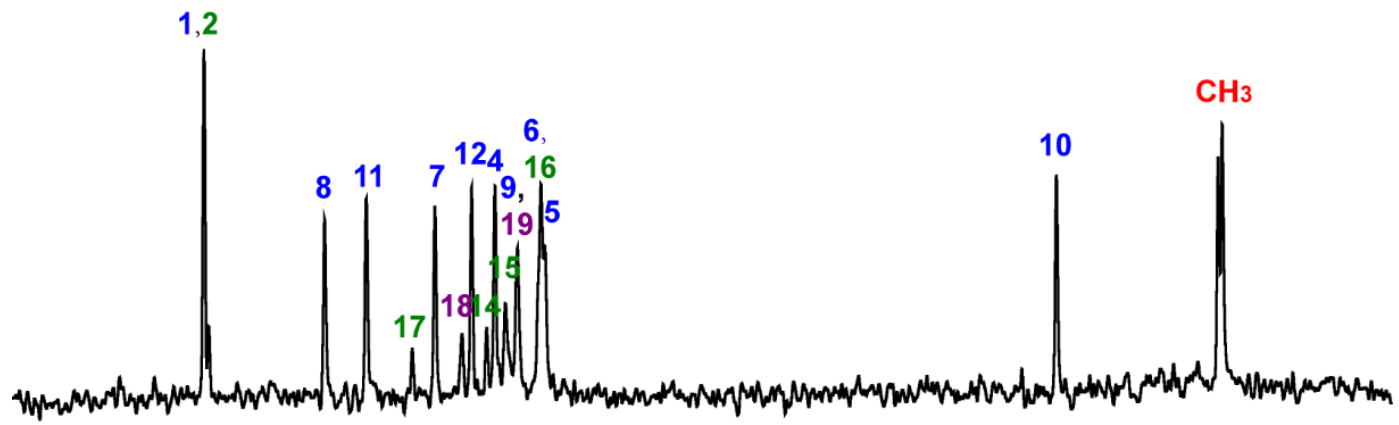
d8-MUF-77-D CPMAS

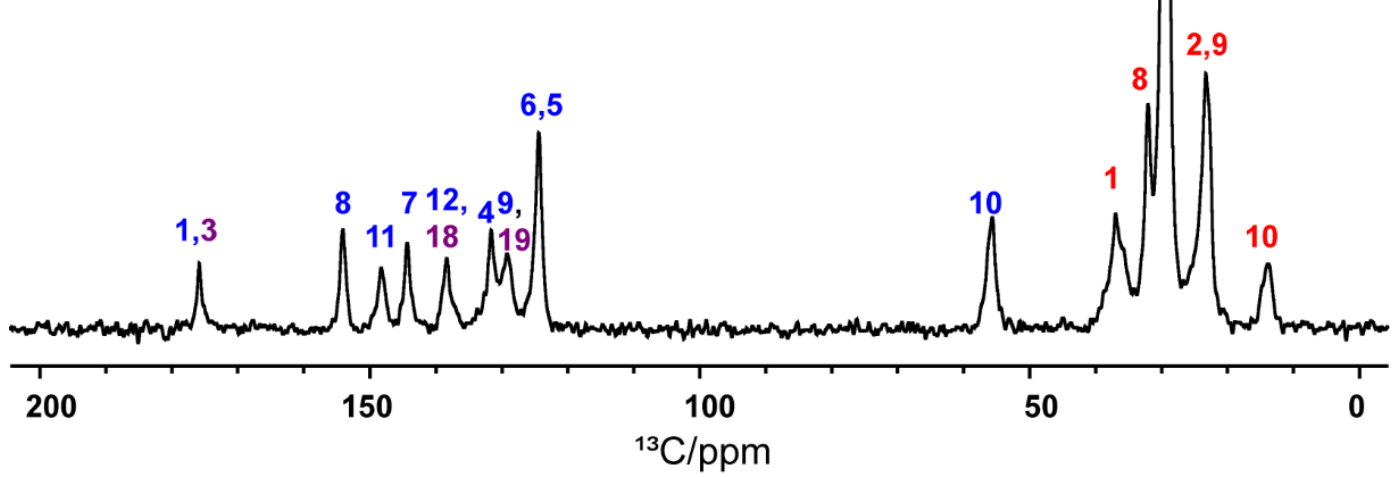

Figure S6. ${ }^{13} \mathrm{C}$ SSNMR spectra of d8-MUF-77-M and d8-MUF-77-D. ${ }^{13} \mathrm{C}$ spectra of d8-MUF-77-

M were obtained by quantitative direct polarization with high power decoupling (HPDEC) using $60 \mathrm{~s}$ recycle delay in which all ${ }^{13} \mathrm{C}$ signals are present. ${ }^{13} \mathrm{C}$ spectra of d8-MUF-77-D was obtained by cross polarization (CPMAS) in which the signals of d8-bpdc are absent. 


\subsection{Studies of Dynamics and Host-Guest Interactions}
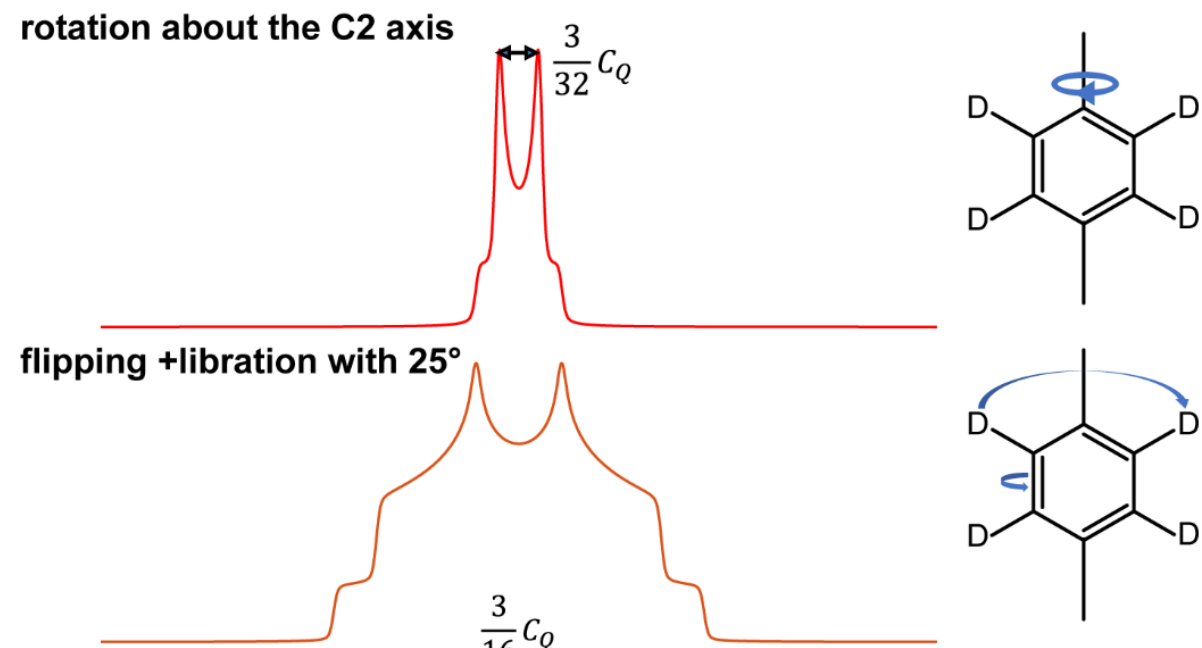<smiles>[2H]c1c([2H])c(CC)c(C)c([2H])c1C</smiles>

$180^{\circ}$ flipping<smiles>[2H]c1c(C)c(C)c([2H])c(CC)c1C</smiles>

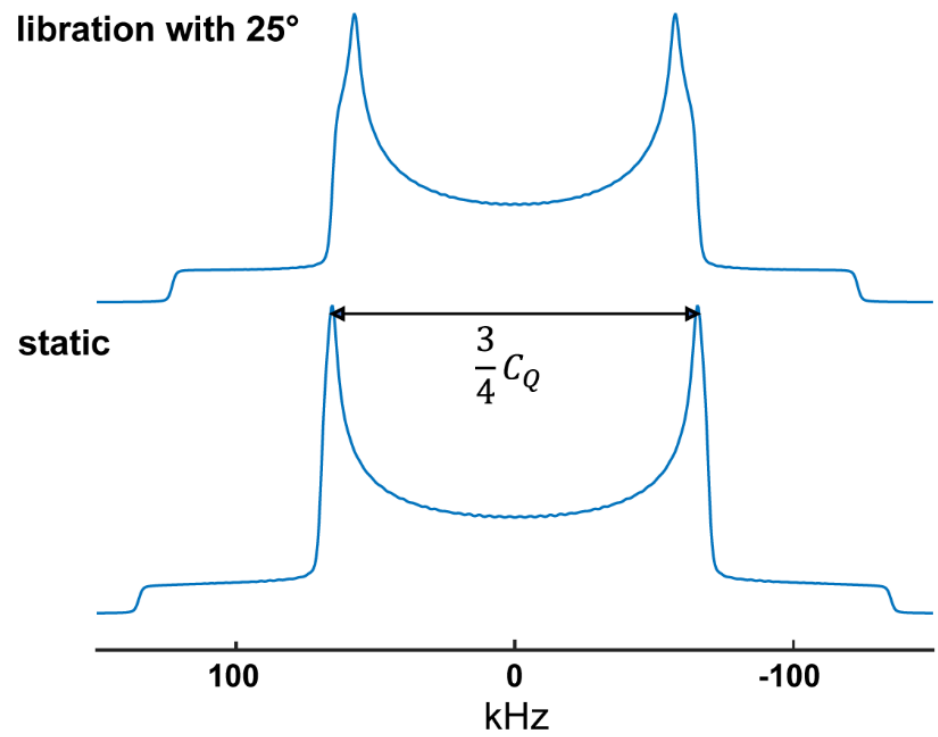<smiles>[2H]c1c([2H])c(C)c([2H])c([2H])c1C</smiles>

Figure S7. Simulated ${ }^{2} \mathrm{H}$ lineshapes for corresponding motion models of the bdc benzene ring.

The lineshape is simulated with a jump rate under fast limit. The widths of the splitting and the shoulder of the lineshape are related to the $\mathrm{C}_{\mathrm{Q}}$ and dynamics of ${ }^{2} \mathrm{H}$. 
a.

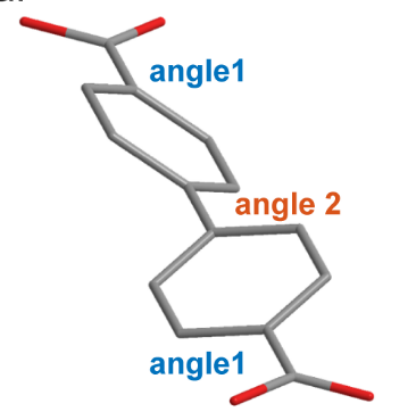

angle1:

O-C-C(Ar)-C(Ar)

angle2:

$C\left(A r_{1}\right)-C\left(A r_{1}\right)-C\left(A r_{2}\right)-C\left(A r_{2}\right)$ b.

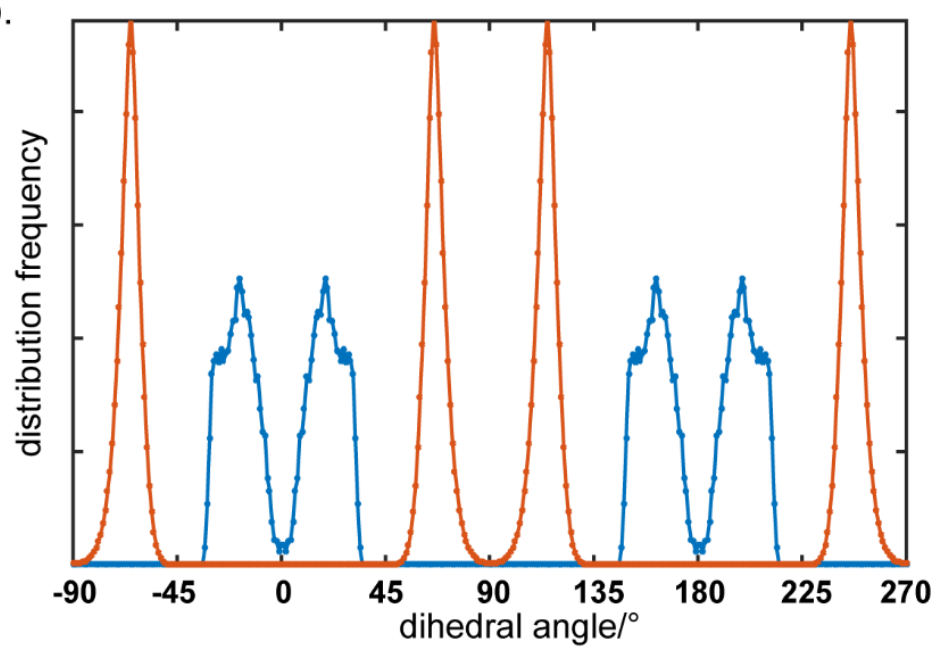

Figure S8. MD simulations of dihedral angle distribution in unloaded and methanol-loaded MUF77-M and MUF-77-D. (a) Illustration of the two kinds of dihedral angle in bpdc ligands: the dihedral angle between phenyl plane and COO plane (angle 1) and the dihedral angle between the two phenyl planes (angle 2). (b) The computational dihedral distribution of bpdc in MUF-77-M. Blue: angle 1; Orange: angle 2 . The angular distributions have a periodicity of $180^{\circ}$ and also have local mirror symmetries separated by about $50^{\circ}$. It is consistent with the bimodal motion $\left(180^{\circ}\right.$ flipping and libration with the angle around $\pm 25^{\circ}$. 


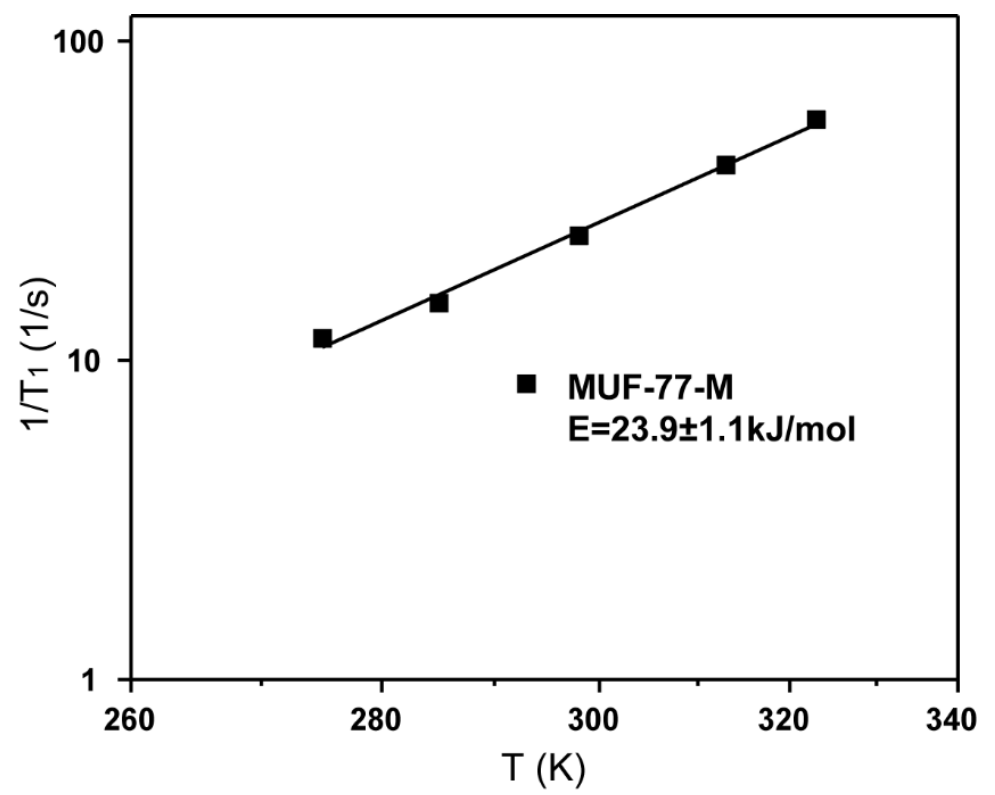

Figure S9. The Arrhenius plot and the calculated activation energy of $180^{\circ}$ flip of d8-bpdc in MUF-77-M from ${ }^{2} \mathrm{H}$ spin-lattice relaxation time $\left(\mathrm{T}_{1}\right)$. E is calculated from the formula $\ln \frac{1}{T_{1}}=$ $-\frac{E_{a}}{R T}+\ln A$. The resultant activation energy is consistent with the value calculated from the jump rates. The error bars are too small compared to the data points. 


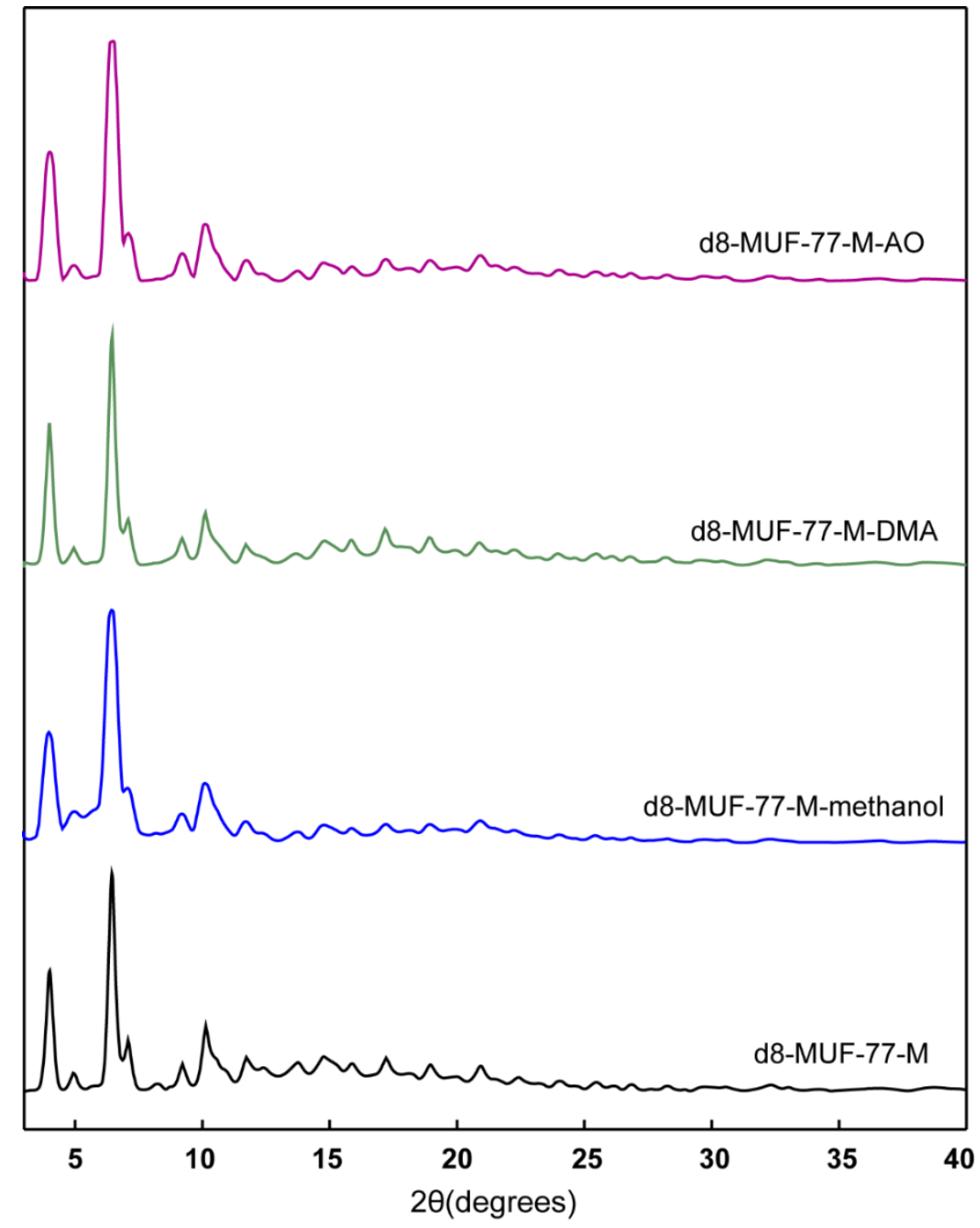

Figure S10. PXRD patterns of pristine d8-MUF-77-M, and d8-MUF-77-M loaded with methanol (blue), DMA (green) and AO (purple). No significant change in PXRD patterns appears after guest adsorption, suggesting that the crystal lattice remains undisturbed. 
a.

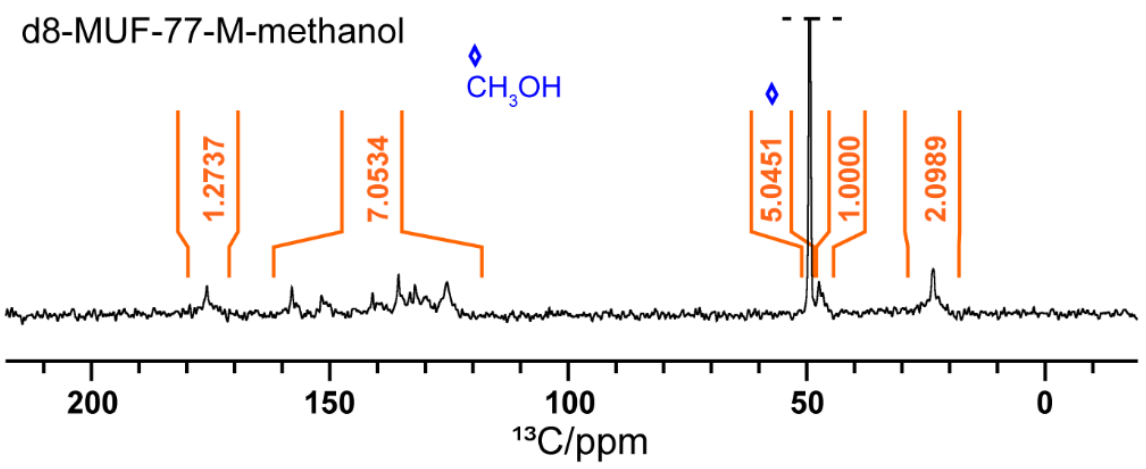

b.

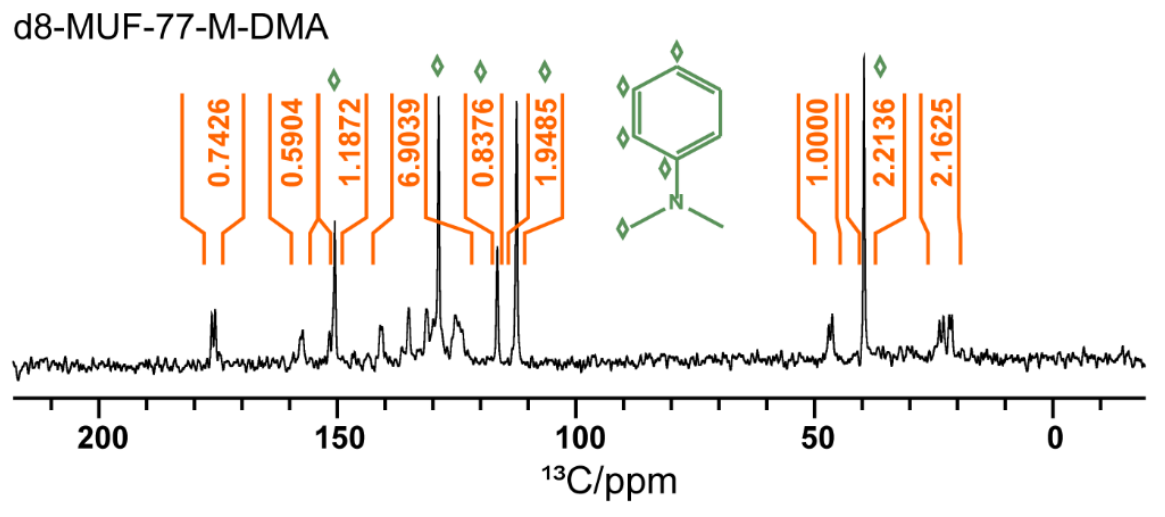

C.

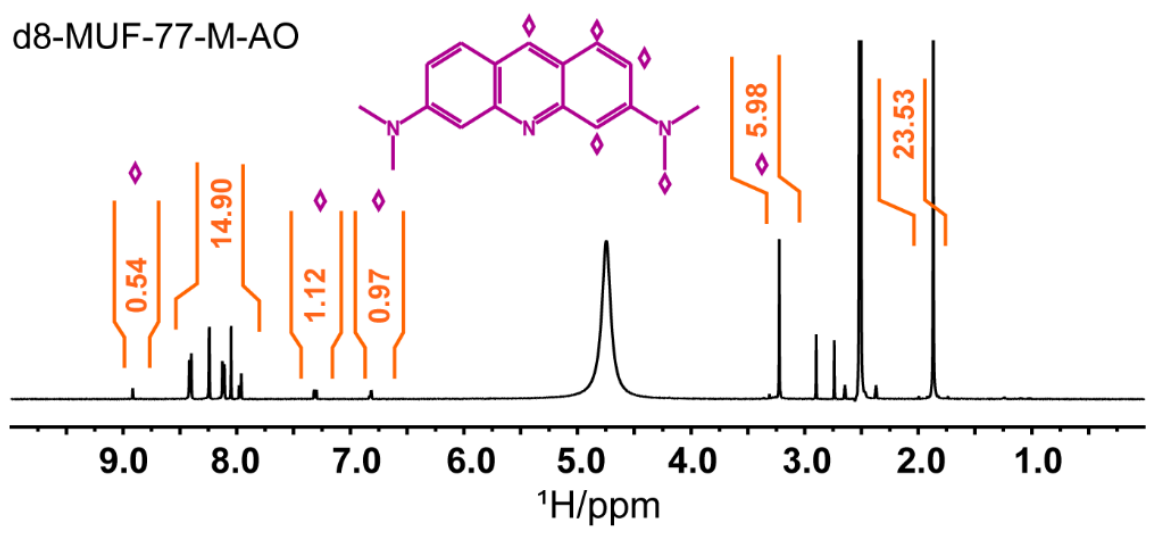

Figure S11. Quantitative direct polarization ${ }^{13} \mathrm{C}$ spectra of (a) d8-MUF-77-M loaded with methanol, (b) d8-MUF-77-M loaded with DMA. (c) Quantitative direct polarization ${ }^{1} \mathrm{H}$ spectra of AO-loaded d8-MUF-77-M dissolved in DCl/dimethylsulfoxide-d6 solvent. The loading concentrations, which means the amounts of adsorbates in every $\left[\mathrm{Zn}_{4} \mathrm{O}(\mathrm{hmtt})_{4 / 3}(\mathrm{bpdc})_{1 / 2}(\mathrm{bdc})_{1 / 2}\right.$, are calculated by the ratios of corresponding signals. Me: conc. $=\frac{A r e a_{M e-C H 3}}{A r e a_{M U F-C H 3}} \times 8=19.2$; DMA: conc. $=\frac{\text { Area }_{D M A-C H 3}}{\text { Area }_{M U F-C H 3}} \times 4=4.1 ; \mathrm{AO}:$ conc. $=\frac{\text { Area }_{A O-C H 3}}{\text { Area }_{M U F-C H 3}} \times 2=0.5$. 
Table S2. The peak widths of ${ }^{13} \mathrm{C}$ CPMAS for d8-MUF-77-M samples.

\begin{tabular}{|c|c|c|c|c|c|c|c|}
\hline \multicolumn{2}{|c|}{ d8-MUF-77-M } & \multicolumn{2}{|c|}{ Methanol loaded } & \multicolumn{2}{|c|}{ DMA loaded } & \multicolumn{2}{|c|}{ AO loaded } \\
\hline $\begin{array}{l}\text { Chemical } \\
\text { shift/ppm }\end{array}$ & $\begin{array}{c}\text { Peak } \\
\text { width } / \mathrm{Hz}\end{array}$ & $\begin{array}{l}\text { Chemical } \\
\text { shift/ppm }\end{array}$ & $\begin{array}{c}\text { Peak } \\
\text { width } / \mathrm{Hz}\end{array}$ & $\begin{array}{l}\text { Chemical } \\
\text { shift/ppm }\end{array}$ & $\begin{array}{c}\text { Peak } \\
\text { width } / \mathrm{Hz}\end{array}$ & $\begin{array}{l}\text { Chemical } \\
\text { shift/ppm }\end{array}$ & $\begin{array}{c}\text { Peak } \\
\text { width } / \mathrm{Hz}\end{array}$ \\
\hline 21.3 & 42.5 & 23.3 & 42.1 & 21.2 & 37.5 & 21.3 & 41.1 \\
\hline \multirow[t]{3}{*}{21.9} & 38.9 & 23.7 & 41.0 & 21.8 & 39.7 & 21.9 & 42.3 \\
\hline & & 23.1 & 288.4 & 23.0 & 43.4 & 23.0 & 250.1 \\
\hline & & & & 23.8 & 42.7 & 24.9 & 250.9 \\
\hline \multirow[t]{2}{*}{46.4} & 44.9 & 46.6 & 145.1 & 46.2 & 53.0 & 46.4 & 80.0 \\
\hline & & 47.4 & 59.8 & 46.9 & 53.7 & 47.3 & 149.8 \\
\hline \multirow[t]{2}{*}{124.0} & 61.1 & 124.5 & 127.3 & 123.9 & 61.1 & 123.9 & 69.7 \\
\hline & & 123.5 & 253.1 & & & & \\
\hline \multirow[t]{2}{*}{124.6} & 55.9 & 125.5 & 79.1 & 124.4 & 54.6 & 124.5 & 70.0 \\
\hline & & 126.9 & 142.2 & 125.3 & 66.3 & 124.8 & 360.1 \\
\hline \multirow[t]{2}{*}{128.2} & 59.5 & 129.8 & 83.1 & 128.1 & 55.3 & 128.1 & 81.4 \\
\hline & & 128.6 & 134.0 & 129.9 & 71.6 & 129.7 & 330.1 \\
\hline \multirow[t]{2}{*}{131.6} & 48.9 & 132.0 & 69.3 & 131.5 & 54.2 & 131.6 & 69.9 \\
\hline & & 130.9 & 87.0 & 131.1 & 59.5 & & \\
\hline \multirow[t]{2}{*}{135.1} & 44.8 & 135.5 & 76.9 & 135.1 & 63.1 & 135.1 & 70.0 \\
\hline & & 133.5 & 197.9 & & & 134.2 & 240.1 \\
\hline \multirow[t]{3}{*}{140.7} & 45.3 & 140.9 & 51.1 & 140.5 & 50.1 & 140.6 & 71.9 \\
\hline & & 140.0 & 99.3 & 141.1 & 45.2 & 139.1 & 262.0 \\
\hline & & 138.9 & 138.9 & & & & \\
\hline \multirow[t]{2}{*}{151.1} & 46.9 & 151.8 & 45.2 & 150.9 & 52.6 & 151.0 & 55.0 \\
\hline & & 150.6 & 157.0 & 151.7 & 41.5 & 150.3 & 200.1 \\
\hline \multirow[t]{2}{*}{157.4} & 46.2 & 157.9 & 52.0 & 157.3 & 52.0 & 157.4 & 66.3 \\
\hline & & 157.1 & 169.9 & 157.8 & 45.6 & 156.5 & 220.5 \\
\hline \multirow[t]{2}{*}{175.8} & 45.2 & 175.8 & 50.1 & 175.6 & 41.4 & 175.7 & 50.0 \\
\hline & & 174.9 & 201.2 & 176.3 & 38.5 & 174.7 & 290.0 \\
\hline 136.6 & 56.0 & 137.3 & 67.0 & 136.4 & 51.9 & 136.6 & 110.2 \\
\hline 175.0 & 43.0 & & & 174.9 & 34.1 & & \\
\hline
\end{tabular}




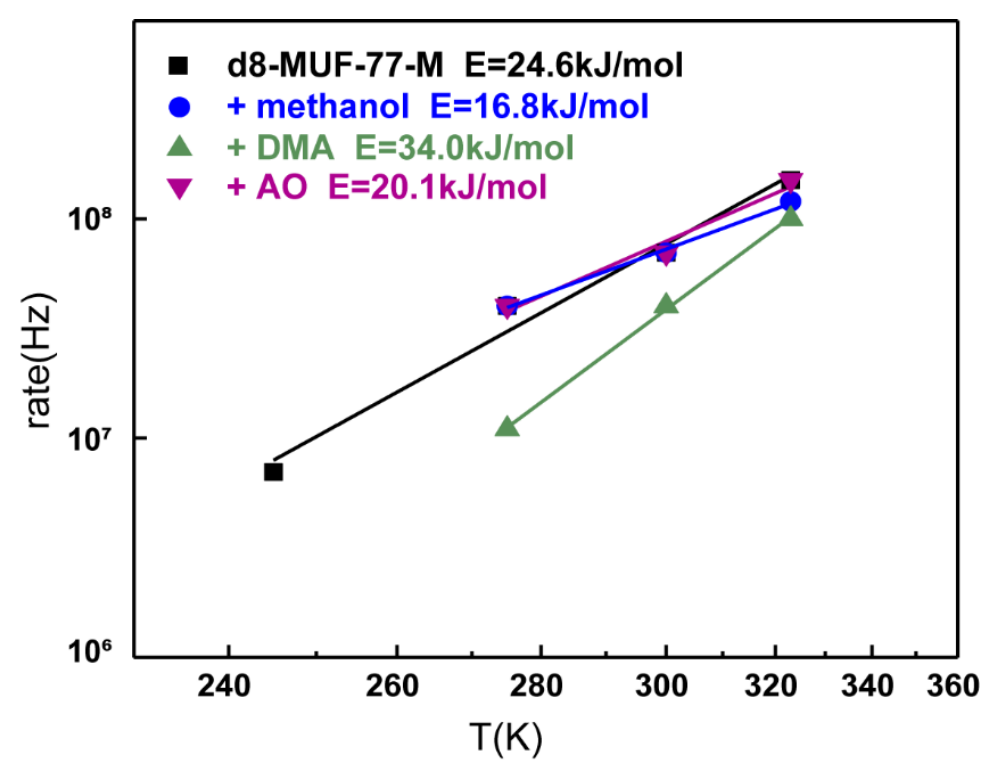

Figure S12. The Arrhenius plot of simulated flipping rate of pristine d8-MUF-77-M (black) and d8-MUF-M loaded with methanol (blue), DMA (green) and AO (purple). $E_{a}$ is the activation energy for the dynamic motion in the Arrhenius equation: $\ln ($ rate $)=-\frac{E_{a}}{R T}+\ln A$. The activation energy of bpdc flipping in DMA-loaded MUF-77-M $(\sim 34 \mathrm{~kJ} / \mathrm{mol})$ is greater than that in pristine MUF-77-M ( $\sim 25 \mathrm{~kJ} / \mathrm{mol})$; while the activation energy of bpdc flipping (considering only the bimodal motion) in methanol or AO-loaded MUF-77-M (about $16 \sim 20 \mathrm{~kJ} / \mathrm{mol}$ ) is about the same as that in pristine MOF. This suggests that the adsorption of DMA affects the bpdc ligands more uniformly than the adsorption of methanol and AO. 
a.

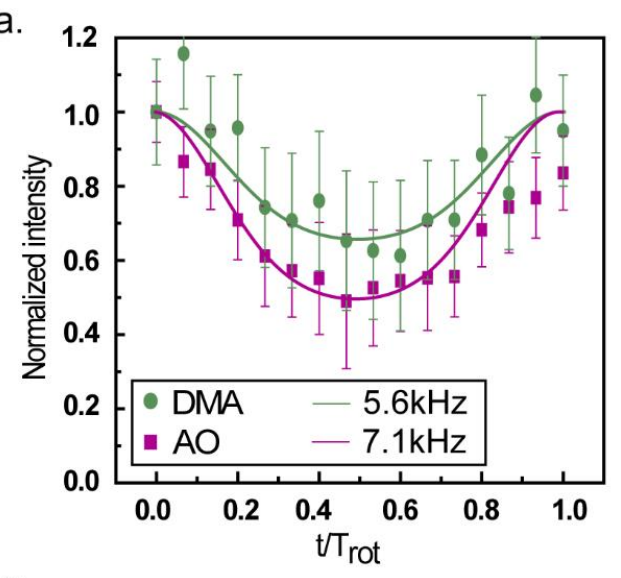

c.

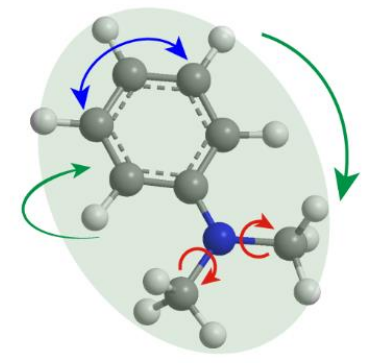

b.
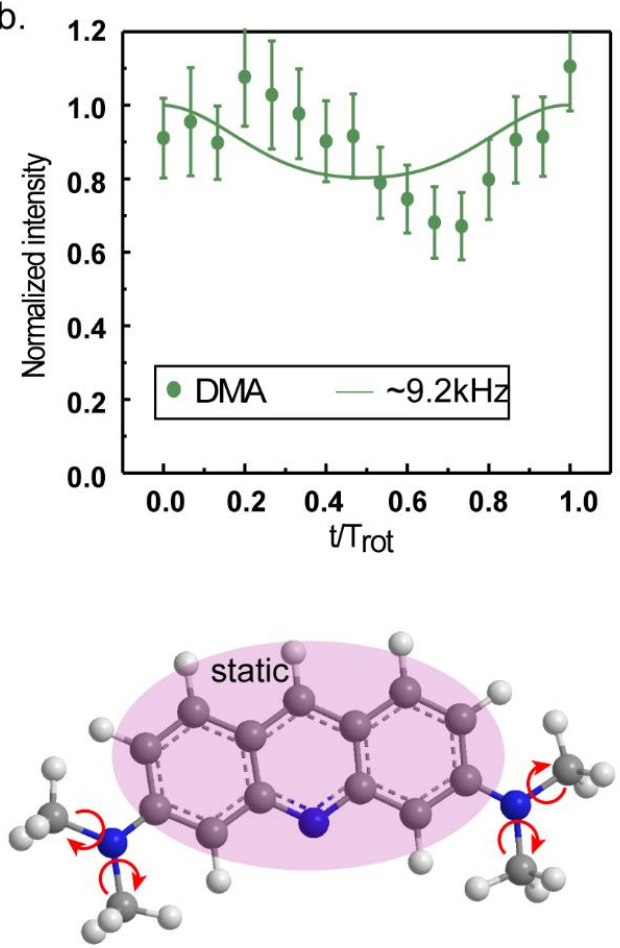

Figure S13. (a) The DIPSHIFT curves of the methyl groups of DMA (green) and AO (purple) absorbed in MUF-77-M. (b)The DIPSHIFT curves of the C-H group on the phenyl ring of DMA (green)absorbed in MUF-77-M. The dipolar constants for the simulated curves are derived according to the equations in ref. 19. (c) The possible dynamic modes of DMA and AO in MUF77-M. The reduced C-H dipolar couplings on the phenyl group compared to the static C-H dipolar coupling $(\sim 22 \mathrm{kHz})$ indicates that the DMA molecule undergoes restricted tumbling as a whole., The adsorbed AO molecule is rigid except the uniaxial rotation of methyl group. 


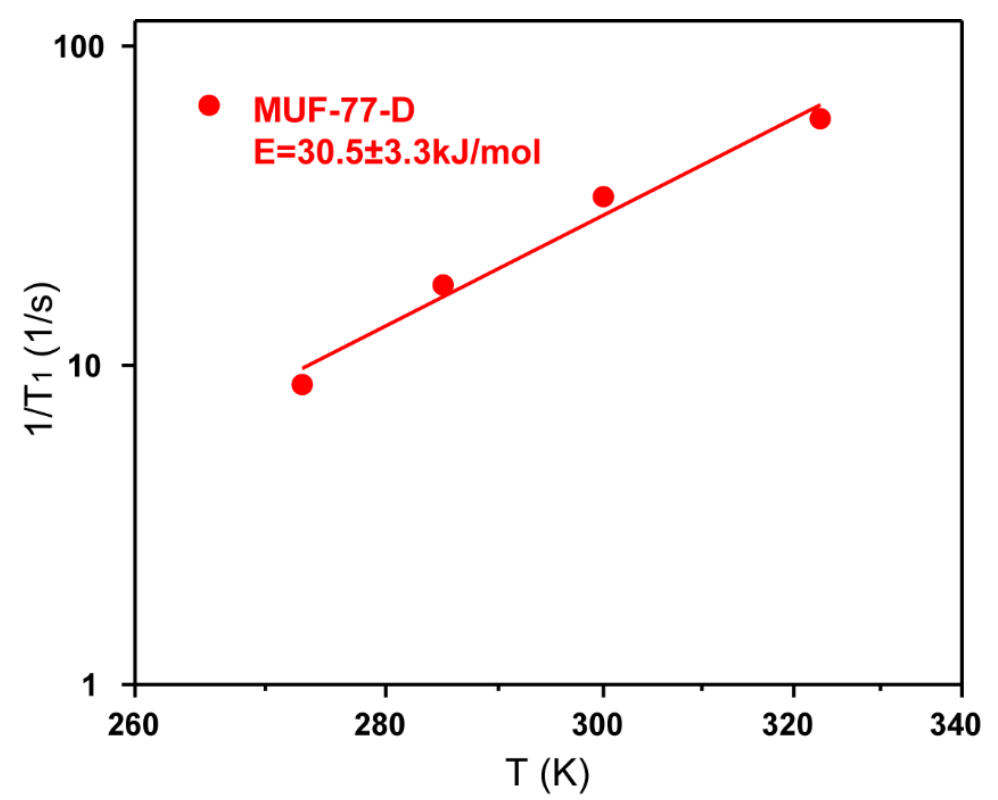

Figure S14. The Arrhenius plot and the calculated activation energy of d8-bpdc of MUF-77-D from ${ }^{2} \mathrm{H}$ spin-lattice relaxation time $\left(\mathrm{T}_{1}\right)$. The resultant activation energy is a little higher than the rotational barrier calculated from the $180^{\circ}$ flipping motion, indicating that the minor part of hindered vibration impacts the average correlation time of the bulk d8-bpdc. The error bars are too small compared to the data points. 
a.

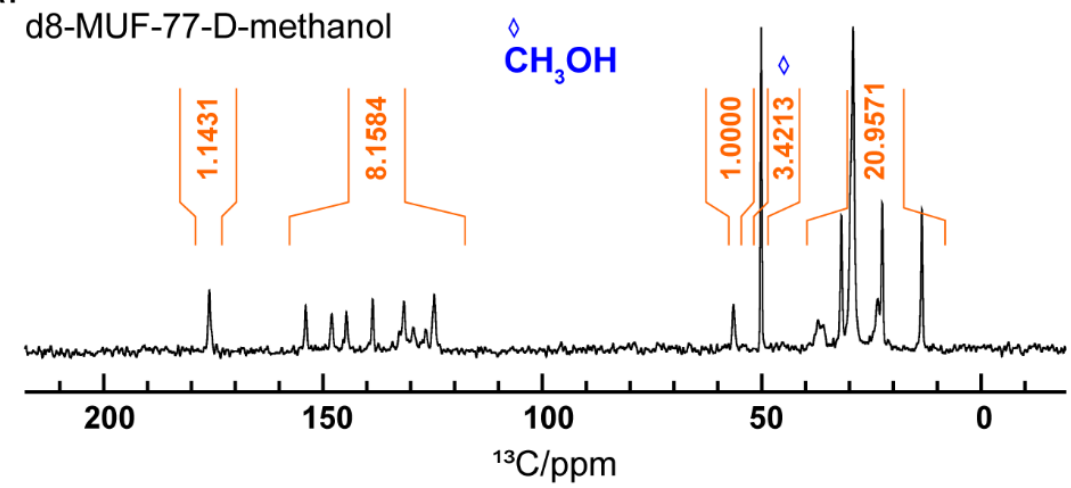

b.

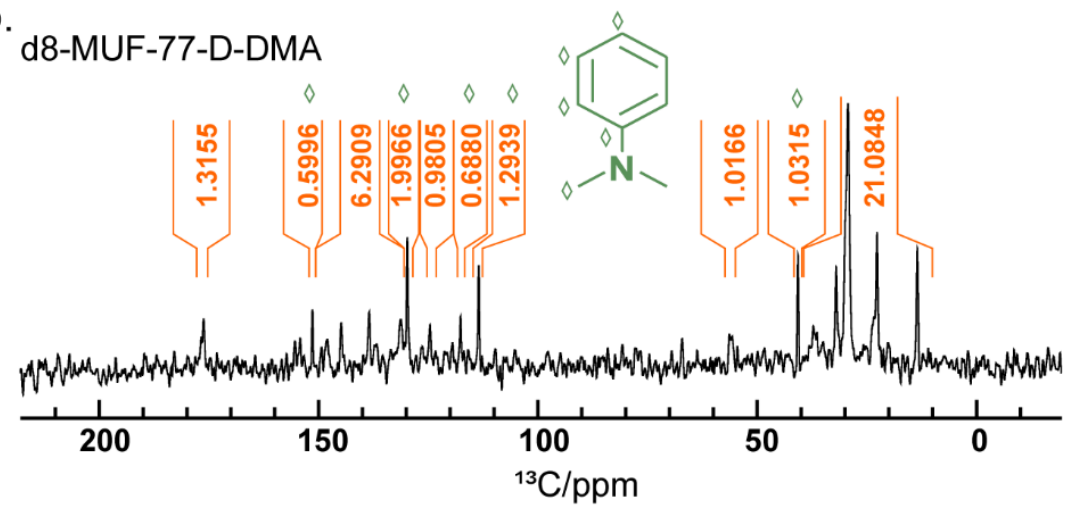

Figure S15. Quantitative direct polarization ${ }^{13} \mathrm{C}$ spectra of d8-MUF-77-D loaded with (a) methanol and (b) DMA. The loading concentrations are calculated by the ratios of corresponding signal. Me: conc. $=\frac{\text { Area }_{M e-C H 3}}{\text { Area }_{M U F-C 10 H 21}} \times 80=13.1$; DMA: conc. $=\frac{\text { Area }_{D M A-C H 3}}{\text { Area }_{M U F-C 10 H 21}} \times 40=2.0$. 
Table S3. The peak widths of ${ }^{13} \mathrm{C}$ CPMAS d8-MUF-77-D samples.

\begin{tabular}{cccccc}
\hline \multicolumn{2}{c}{ d8-MUF-77-D } & \multicolumn{2}{c}{ Methanol loaded } & \multicolumn{2}{c}{ DMA loaded } \\
$\begin{array}{c}\text { Chemical } \\
\text { shift/ppm }\end{array}$ & $\begin{array}{c}\text { Peak } \\
\text { width/Hz }\end{array}$ & $\begin{array}{c}\text { Chemical } \\
\text { shift/ppm }\end{array}$ & $\begin{array}{c}\text { Peak } \\
\text { width/Hz }\end{array}$ & $\begin{array}{c}\text { Chemical } \\
\text { shift/ppm }\end{array}$ & $\begin{array}{c}\text { Peak } \\
\text { width/Hz }\end{array}$ \\
\hline 13.8 & 190.3 & 13.5 & 47.0 & 13.3 & 54.6 \\
23.3 & 177.7 & 22.5 & 58.0 & 23.4 & 152.3 \\
& & 23.6 & 102.8 & & \\
29.3 & 156.3 & 29.1 & 98.9 & 29.2 & 103.8 \\
32.0 & 134.3 & 31.9 & 50.5 & 31.9 & 54.4 \\
$37.0 *$ & 250.7 & 37.1 & 187.4 & 35.7 & 268.6 \\
55.6 & 142.3 & 56.3 & 64.8 & 55.9 & 78.3 \\
124.3 & 117.0 & 124.4 & 73.5 & 124.3 & 77.6 \\
129.1 & 209.1 & 129.2 & 95.3 & 129.3 & 122.8 \\
131.5 & 135.3 & 131.6 & 79.3 & 131.4 & 84.4 \\
138.3 & 121.5 & 138.6 & 48.8 & 138.4 & 43.4 \\
144.2 & 93.8 & 144.6 & 56.5 & 144.5 & 55.9 \\
148.2 & 122.5 & 148.0 & 62.8 & 148.0 & 67.5 \\
154.0 & 99.7 & 154.0 & 50.4 & 154.0 & 65.2 \\
175.7 & 99.4 & 175.9 & 55.6 & 176.1 & 59.7 \\
\hline
\end{tabular}




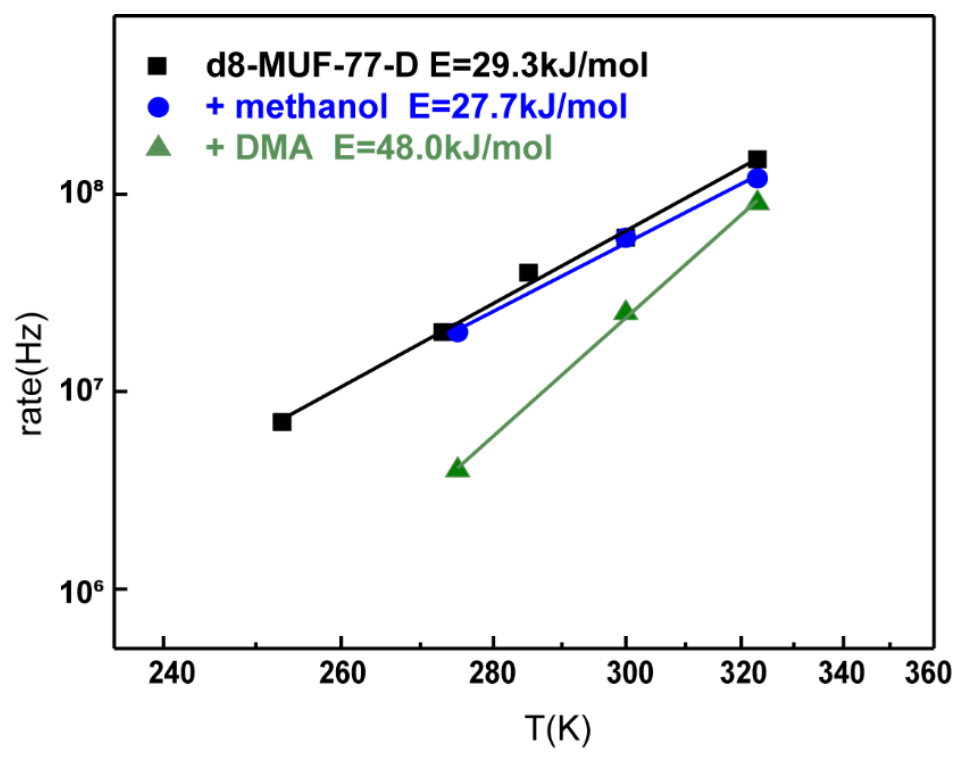

Figure S16. The Arrhenius plot of simulated flipping rate of pristine d8-MUF-77-D (black) and d8-MUF-77-D loaded with methanol (blue) and DMA (green). DMA-loading significantly increases the activation energy of the bimodal flipping motion of bpdc. 


\section{REFERENCES}

(1) Liu, L.; Telfer, S. G., Systematic Ligand Modulation Enhances the Moisture Stability and Gas Sorption Characteristics of Quaternary Metal-Organic Frameworks. J. Am. Chem. Soc. 2015, 137, 3901-3909.

(2) Vold, R. L.; Hoatson, G. L., Effects of Jump Dynamics on Solid State Nuclear Magnetic Resonance Line Shapes and Spin Relaxation Times. J. Magn. Reson. 2009, 198, 57-72.

(3) Li, K.; Emani, P. S.; Ash, J.; Groves, M.; Drobny, G. P., A Study of Phenylalanine SideChain Dynamics in Surface-Adsorbed Peptides Using Solid-State Deuterium Nmr and Rotamer Library Statistics. J. Am. Chem. Soc. 2014, 136, 11402-11411.

(4) Dominguez, Z.; Dang, H.; Strouse, M. J.; Garcia-Garibay, M. A., Molecular "Compasses" and "Gyroscopes." Iii. Dynamics of a Phenylene Rotor and Clathrated Benzene in a Slipping-Gear Crystal Lattice. J. Am. Chem. Soc. 2002, 124, 7719-7727.

(5) Horike, S.; Matsuda, R.; Tanaka, D.; Matsubara, S.; Mizuno, M.; Endo, K.; Kitagawa, S., Dynamic Motion of Building Blocks in Porous Coordination Polymers. Angew. Chem. Int. Ed. 2006, 45, 7226-7230.

(6) Rodriguez-Velamazan, J. A., et al., A Switchable Molecular Rotator: Neutron Spectroscopy Study on a Polymeric Spin-Crossover Compound. J. Am. Chem. Soc. 2012, 134, 5083-5089.

(7) Barnes, R. G.; Bloom, J. W., Measurement of the Quadrupole Coupling Constant and Asymmetry Parameter of the Ring Deuterons in Several Deuterated Benzene Derivatives. J. Chem. Phys. 1972, 57, 3082-3086.

(8) Huber, H., Deuterium Quadrupole Coupling Constants. A Theoretical Investigation. J. Chem. Phys. 1985, 83, 4591-4598. 
(9) Millett, F. S.; Dailey, B. P., NMR Determination of Some Deuterium Quadrupole Coupling Constants in Nematic Solutions. J. Chem. Phys. 1972, 56, 3249-3256.

(10) Olympia, P. L.; Wei, I. Y.; Fung, B. M., Deuteron Quadrupole Coupling Constants in Deuterocarbons. J. Chem. Phys. 1969, 51, 1610-1614.

(11) Schmidt-Rohr, K.; Spiess, H. W., Multidimensional Solid-State NMR and Polymers; Elsevier, 2012, pages 126-130.

(12) Van Der Spoel, D.; Lindahl, E.; Hess, B.; Groenhof, G.; Mark, A. E.; Berendsen, H. J., Gromacs: Fast, Flexible, and Free. J. Comput. Chem. 2005, 26, 1701-1718.

(13) Malde, A. K.; Zuo, L.; Breeze, M.; Stroet, M.; Poger, D.; Nair, P. C.; Oostenbrink, C.; Mark, A. E., An Automated Force Field Topology Builder (ATB) and Repository: Version 1.0. J. Chem. Theory Comput. 2011, 7, 4026-4037.

(14) Frisch, M.; Trucks, G.; Schlegel, H.; Scuseria, G.; Robb, M.; Cheeseman, J.; Montgomery Jr, J.; Vreven, T.; Kudin, K.; Burant, J., Gaussian 03, Revision C. 02. Wallingford, Ct: Gaussian. Inc. 2004.

(15) Tafipolsky, M.; Schmid, R., Systematic First Principles Parameterization of Force Fields for Metal-Organic Frameworks Using a Genetic Algorithm Approach. J. Phys. Chem. B 2009, 113, 1341-1352.

(16) Oostenbrink, C.; Villa, A.; Mark, A. E.; Van Gunsteren, W. F., A Biomolecular Force Field Based on the Free Enthalpy of Hydration and Solvation: The Gromos Force-Field Parameter Sets 53A5 and 53A6. J. Comput. Chem. 2004, 25, 1656-1676.

(17) Darden, T.; York, D.; Pedersen, L., Particle Mesh Ewald: An N·Log (N) Method for Ewald Sums in Large Systems. J. Chem. Phys. 1993, 98, 10089-10092.

(18) Berendsen, H. J.; Postma, J. v.; van Gunsteren, W. F.; DiNola, A.; Haak, J. R., Molecular Dynamics with Coupling to an External Bath. J. Chem. Phys. 1984, 81, 3684-3690. 
(19) deAzevedo, E. R.; Saalwachter, K.; Pascui, O.; de Souza, A. A.; Bonagamba, T. J.; Reichert, D., Intermediate Motions as Studied by Solid-State Separated Local Field NMR Experiments. J. Chem. Phys. 2008, 128, 104505. 